Article

\title{
Supramolecular Assemblies from Poly(styrene)-block-poly(4- vinylpyridine) Diblock Copolymers Mixed with 6-Hydroxy-2-naphthoic Acid
}

\author{
Bhavesh Bharatiya, Jean-Marc Schumers, Elio Poggi and Jean-François Gohy * \\ Bio and Soft Matter (BSMA), Institute of Condensed Matter and Nanosciences (IMCN), \\ Catholic University of Louvain (UCL), Place Louis Pasteur 1, Louvain-la-Neuve 1348, Belgium; \\ E-Mails: bhaveshbharatiya@gmail.com (B.B.); jean-marc.schumers@cytec.com (J.-M.S.); \\ elio.poggi@uclouvain.be (E.P.)
}

* Author to whom correspondence should be addressed; E-Mail: jean-francois.gohy@uclouvain.be; Tel.: +32-10-479-269; Fax: +32-10-479-269.

Received: 14 April 2013; in revised form: 22 May 2013 / Accepted: 28 May 2013 /

Published: 5 June 2013

\begin{abstract}
Supramolecular assemblies involving interaction of a small organic molecule, 2-hydroxy-6-Naphthoic acid (HNA), with poly(styrene)-block-poly(4-vinylpyridine) (PS- $b$-P4VP) diblock copolymers are utilized to obtain micellar structures in solution, nanostructured thin films on flat substrates and, finally, nanoporous thin films. The formation of hydrogen bonds between HNA and the poly(4-vinylpyridine) (P4VP) blocks is confirmed by spectroscopic measurements. The accordingly P4VP/HNA hydrogen-bonded complexes are poorly soluble in 1,4-dioxane, resulting in the formation of micellar structures with a P4VP/HNA core and a polystyrene (PS) corona. Those micelles have been spin-coated onto silicon wafers, resulting in nanostructured thin films consisting of P4VP/HNA dot-like features embedded in a PS matrix. The morphology of those films has been tuned by solvent annealing. Selective dissolution of HNA by methanol results in the formation of a nanoporous thin film. The P4VP/HNA nanodomains have been also cross-linked by borax, and the thin films have been further dissolved in a good solvent for PS, leading to micelles with a structure reminiscent of the thin films.
\end{abstract}

Keywords: block copolymers; micelles; thin films; nanoporous materials; hydrogen-bonding; morphology 


\section{Introduction}

Block copolymers are known to self-assemble into structures of nanoscopic length scale, either in a selective solvent for one of the blocks (micellar structures) or in bulk and continue to remain a prominent research topic [1-7]. In the typical application of block copolymers as thin films, the structural behavior could be more complicated compared to the bulk, due to their interactions with the underlying substrate. This class of ordered materials is applicable to the fields of, e.g., lithography, surface patterning, semiconducting nanomaterials and biosensing devices [8-13].

Association of small organic molecules with one of the polymer blocks of the starting block copolymers via weak forces of attractions, like electrostatic interactions, van der Waals forces and hydrogen bonding, has become a widely adopted approach for the fine-tuning of the block copolymer morphology in solution [14], bulk [15,16] and in thin films [17-27]. In a further step, the interacting small molecules can be removed by dissolution in a selective solvent, and nanoporous materials can be obtained $[17,18]$.

A very important issue whenever block copolymer self-assemblies are concerned is the equilibrium or non-equilibrium state of the nanostructures obtained after the self-assembly process. Due to the intrinsic nature of polymer chains, the obtained nanostructures (be in solution, bulk or thin films) may be kinetically trapped non-equilibrium systems, since the constituent polymer chains may not be able to relax towards their equilibrium conformation. The possibility to freeze-in non-equilibrium structures in block copolymer self-assemblies significantly broadens the range of achievable nanostructures. Nevertheless, this could be also a hint towards the formation of reproducible structures. As typical examples, the lateral ordering and morphology in block copolymer thin films are highly dependent on the nature of the solvent and on the annealing conditions [28-31].

As far as supramolecular complexes between block copolymers and small organic molecules are concerned, poly(styrene)-block-poly(4-vinylpyridine) (PS-b-P4VP) block copolymers, which are commercially available in a large variety of molar masses, have been widely investigated. The generally adopted strategy involves the interaction between the small organic molecules and 4-vinylpyridine (4VP) groups, leading to the formation of hydrogen bonds. In this way, the introduction of the small molecules can alter the relative volume fraction of the P4VP block, and morphological changes could be induced, leading to a variety of hierarchical assemblies. The most commonly reported small molecules for such supramolecular complexes are 2-(4'-hydroxybenzeneazo)benzoic acid (HABA) and 3-pentadecylphenol (PDP). Tung et al. [21,31] and ten Brinke et al. [32,33] have investigated the structure-within-structure morphologies in thin films involving the interaction of PDP with P4VP. Tung et al. have demonstrated the correlation between the morphology orientation in thin films and the volume fraction of P4VP/PDP block [21]. Ten Brinke and coworkers have reported the terrace formation in thin films comprising P4VP/PDP supramolecular assemblies [32]. Stamm and coworkers realized homogeneous thin films from the supramolecular assembly of PS- $b$-P4VP and HABA [17,18,34-36]. The orientation of the resultant cylindrical morphology could be switched from vertical to horizontal, depending on the solvent conditions. The dip-coated films from PS- $b$-P4VP containing HABA in tetrahydrofuran (THF) showed the presence of mixed vertical and horizontal cylindrical morphologies, while the morphology of the dip-coated thin films from 1,4-dioxane solution 
showed vertical cylinders and that from chloroform showed horizontal cylinders. Nandan et al. have shown morphological changes in the film morphology by changing the P4VP/HABA molar ratio [36].

A few attempts have been made to investigate the interaction of PS- $b$-P4VP diblock copolymers with small organic molecules, other than PDP and HABA. Kuila et al. reported the film morphology from PS- $b$-P4VP and 1-pyrenebutyric acid (PBA), where the cylindrical morphology changed to lamella for the dip-coated films from 1,4-dioxane solution [11]. Prud'homme et al. investigated the thin films from PS- $b$-P4VP and 1,5-dihydroxy naphthalene (DHN) in THF, which led to quasi-hexagonal nodular morphology [19]. The depth of the nanopores obtained after washing out DHN in methanol was a function of the P4VP/DHN molar ratio, and the highest value was measured for the 1:4 ratio. Recently, Bazuin et al. used naphthol and naphthoic acid to induce morphology changes by tuning the interactions of those small molecules with P4VP blocks [37-39]. It was concluded that for P4VP/small molecule molar ratio of $1: 1$, the small molecule with hydroxyl functionality led to dot-like morphology, while that with carboxylic acids essentially showed stripe (horizontal cylinders) morphology. It was also demonstrated than other factors, like the dip-coating rate, the concentration and the P4VP/small molecule molar ratio, could also control the dot or stripe morphology. Obviously, non-equilibrium structures are mainly observed from those previously investigated PS- $b$-P4VP/small molecules complexes that can be further tuned by applying proper experimental conditions.

In this paper, we investigate supramolecular non-covalent complexes from PS- $b$-P4VP diblock copolymers and 6-hydroxy-2-naphthoic acid (HNA) in 1,4-dioxane. The aim is to investigate the effect of the two functional groups (hydroxyl and carboxylic acid groups) in the used small molecule on the self-assembly process in solution (micelles) and in thin-films. Our attempts will broaden the scope of using such non-equilibrium supramolecular assemblies for application in nanoscience and nanotechnology.

\section{Experimental Section}

\subsection{Materials}

A poly(styrene)-block-poly(4-vinylpyridine) PS- $b$-P4VP block copolymer $\left(M_{\mathrm{w}} / M_{\mathrm{n}}=1.12\right)$ with a PS molar mass of 57,500 $\mathrm{g} \cdot \mathrm{mol}^{-1}$ and a P4VP molar mass of 18,500 $\mathrm{g} \cdot \mathrm{mol}^{-1}$ was purchased from Polymer Source Inc. (Dorval, Quebec, Canada) and used as received. The small organic molecule 6-hydroxy-2-naphthoic acid (HNA, structure depicted in Scheme 1) and borax $\left(\mathrm{Na}_{2} \mathrm{~B}_{4} \mathrm{O}_{7}\right)$ were purchased from Sigma-Aldrich. 1,4-dioxane, tetrahydrofuran (THF) and methanol were purchased from Sigma-Aldrich and filtered through $0.2 \mu \mathrm{m}$ filters prior to the use.

Scheme 1. Chemical structure of 6-hydroxy-2-naphthoic acid (HNA).<smiles>O=C(O)c1ccc2cc(O)ccc2c1</smiles> 


\subsection{Preparation of Samples}

Accurately weighted required amounts of PS- $b$-P4VP diblock copolymer and HNA were dissolved separately in 1,4-dioxane under constant stirring for $2 \mathrm{~h}$. Both solutions were then heated in a sonication bath at $80{ }^{\circ} \mathrm{C}$ for $3 \mathrm{~h}$. The copolymer solution was then dropwise added to the solution of HNA under sonication at $80{ }^{\circ} \mathrm{C}$ in order to break the intra- or inter-molecular H-bonds formed in the HNA solution. The accordingly obtained PS- $b$-P4VP/HNA mixtures were allowed to cool to room temperature and kept overnight. The solutions were filtered with $0.2 \mu \mathrm{m}$ PTFE filters prior to dynamic light scattering analysis or spin-coating. For the preparation of thin films, silicon substrates were cleaned by a piranha solution $\left(\mathrm{H}_{2} \mathrm{SO}_{4} 98 \% / \mathrm{H}_{2} \mathrm{O}_{2} 30 \%, 3: 1\right)$ for $90 \mathrm{~min}$ before being rinsed with ultra-pure water. The substrates were then dried with the spin-coater at a velocity of $4000 \mathrm{rpm}$ for $20 \mathrm{~s}$. Filtered solutions of PS- $b$-P4VP/HNA mixtures in 1,4-dioxane were then spin-coated onto these substrates at $2000 \mathrm{rpm}$ during $40 \mathrm{~s}$. The thickness of the films was controlled by the solution concentration and was evaluated by ellipsometry (SE 800 PV ellipsometer from Sentech, Cambridge, UK). The average thicknesses of the films were between 45 and $55 \mathrm{~nm}$. The thin films were then annealed in 1,4-dioxane or THF vapors for $24 \mathrm{~h}$ in a sealed glass chamber. Two $25 \mathrm{~mL}$ beakers containing $10 \mathrm{~mL}$ of 1,4-dioxane were placed in this chamber together with the thin films. To cross-link the P4VP/HNA nanodomains by borax, the thin films were immersed for $4 \mathrm{~h}$ in a slightly acidic $\mathrm{Na}_{2} \mathrm{~B}_{4} \mathrm{O}_{7}$ aqueous solution at $\mathrm{pH}=5$ (in order to favor penetration inside the P4VP/HNA nanodomains) and $10 \mathrm{~g} / \mathrm{L}$ and then rinsed with pure water. The cross-linked films were detached from the substrate by immersing them in 1,4-dioxane for three days. To remove HNA molecules, the thin films were immersed in methanol for $30 \mathrm{~min}$, dried under a nitrogen flow and then kept in a vacuum oven at $80{ }^{\circ} \mathrm{C}$ for 5 min to ensure complete methanol evaporation.

\subsection{Dynamic Light Scattering (DLS) Measurements}

The solutions containing the PS- $b$-P4VP/HNA mixtures were filtered through $0.2 \mu \mathrm{m}$ filters prior to the measurements. DLS experiments were performed on a Malvern CGS-3 apparatus equipped with a He-Ne laser with a wavelength of $633 \mathrm{~nm}$. The measurements were performed in 1,4-dioxane at a $90^{\circ}$ angle and at a concentration of $1 \mathrm{wt} \%$. The data were analyzed using the constrained regularization method for inverting data (CONTIN) method, which is based on an inverse-Laplace transformation of the data. The polydispersity index (PDI) of the micelles was estimated from the $\Gamma_{2} / \Gamma_{1}^{2}$ ratio in which $\Gamma_{1}$ and $\Gamma_{2}$ represent the first and second order moments calculated from the cumulants method.

\subsection{Transmission Electron Microscopy (TEM) Measurements}

TEM images were recorded on a LEO 922 microscope (Carl Zeiss, Jena, Germany), operating at $200 \mathrm{kV}$ accelerating voltage in bright field mode. The samples were prepared by drop casting of a pre-diluted micellar solution $(0.05 \mathrm{~g} / \mathrm{L})$ on a TEM carbon-coated copper grid (200 mesh). The samples were then dried one day at room temperature. The micelles were stained for 20 min by $\mathrm{RuO}_{4}$ vapors, obtained upon mixing $\mathrm{RuCl}_{3}$ with $\mathrm{NaClO}$ at a concentration of $2 \% \mathrm{w} / \mathrm{v}$. 


\subsection{Fourier Transform Infrared Spectroscopy (FTIR) Measurements}

Infrared (IR) spectra were recorded in reflectance mode on thin films deposited onto silicon wafers with a Shimadzu FTIR-8400S FT-IR spectrometer (Shimadzu Benelux, Antwerpen, Belgium). Two weight percent solutions of PS- $b$-P4VP/HNA mixtures were spin coated onto silicon wafers. The average thickness of the films was $\approx 90 \mathrm{~nm}$, as measured by ellipsometry.

\subsection{Atomic Force Microscopy (AFM) Measurements}

Atomic force microscopy (AFM) was performed on a Digital Instruments Nanoscope IV scanning force microscope operated in tapping mode using NCL cantilevers $(\mathrm{Si}, 48 \mathrm{~N} / \mathrm{m}, 330 \mathrm{kHz}$, Nanosensors, Neuchatel, Switzerland).

\section{Results and Discussion}

\subsection{Micellar Structures in 1,4-Dioxane}

Complexation through non-covalent interactions of one of the block of a block copolymer with small organic molecules may lead to the insolubilization of the complexed polymer block and its further aggregation into a micellar core. In this scenario, the accordingly formed micellar core is stabilized by coronal chains from the remaining uncomplexed soluble blocks of the starting block copolymer. This strategy has been widely used to form electrostatic complexes from a polyelectrolytic block and oppositely charged surfactant molecules, as reported by several groups in the mid-1990s [39-41].

In the present study, we aim at forming micelles in 1,4-dioxane from mixtures of a PS- $b$-P4VP diblock copolymer with HNA. The expected structure for those micelles is an insoluble P4VP/HNA core surrounded by PS coronal chains. Indeed, it has been reported that PS is soluble in 1,4-dioxane, while P4VP is not [31]. Therefore, the starting PS-b-P4VP copolymer already formed micellar structures, as confirmed by DLS and TEM analysis (Figure 1a). The DLS results showed micelles with an average hydrodynamic radius $\left(R_{\mathrm{h}}\right)$ of $40.8 \pm 0.7 \mathrm{~nm}$ at a concentration of $1 \mathrm{wt} \%$ and a relatively narrow PDI of 0.11 . The measurements were extended at different angles and concentrations, and it was confirmed that $R_{\mathrm{h}}$ was almost independent of the concentration (at least in the investigated range from 0.2 to $2 \mathrm{wt} \%$ ) and of the angle of measurement (data not shown). This suggests the formation of spherical Micelles. In a second step, the solutions containing the PS- $b$-P4VP/HNA mixtures were investigated in 1,4-dioxane. DLS revealed the formation of micelles with a $R_{\mathrm{h}}$ of $41.5 \pm 0.9 \mathrm{~nm}$ and a PDI of 0.10 for the PS- $b$-P4VP/HNA mixture containing a 4VP/HNA molar ratio of $1: 1$. The obtained values confirm identical characteristic features within experimental uncertainty for PS- $b$-P4VP micelles with or without added HNA. Again, the typical size of those micelles did not change whenever the angle of measurement, the concentration and the 4VP/HNA molar ratio (1:0.25, 1:0.5, 1:0.75 and 1:1 4VP/HNA molar ratios were investigated) were changed. This suggests, again, that spherical micelles are formed for the PS- $b$-P4VP/HNA mixtures. In a recent publication, Bazuin and co-workers have investigated the formation of micelles from PS- $b$-P4VP diblock copolymers mixed with naphthol and naphthoic acid in THF [37]. They also observed the formation of spherical micelles showing identical characteristic features with or without small molecules added to the PS- $b$-P4VP 
copolymers. Those authors concluded that there was the formation of highly swollen P4VP cores for the PS- $b$-P4VP micelles in THF. Indeed, the comparison of the $R_{\mathrm{h}}$ of those micelles to their radius of gyration $\left(R_{\mathrm{g}}\right)$ as determined by static light scattering led to $R_{\mathrm{g}} / R_{\mathrm{h}}$ ratio always below 0.77 , thus indicating soft spherical micelles rather than hard spheres [37]. The fact that no change in the micellar characteristic features was observed upon addition of small molecules to the initial PS- $b$-P4VP micelles was attributed to the fact that the small molecules essentially remained in solution because of their interaction through H-bonding with THF molecules and had therefore no reason to be localized into the micellar cores. The same situation could prevail here for the PS- $b$-P4VP/HNA mixtures in 1,4-dioxane. TEM imaging confirms the formation of spherical micelles from PS- $b$-P4VP/HNA mixtures (Figure 1b,c). Moreover, the characteristic sizes of those micelles (diameters around 15-20 nm) dried on the TEM grid are significantly smaller compared to the $R_{\mathrm{h}}$ determined in solution. This suggests that the PS- $b$-P4VP and PS- $b$-P4VP/HNA micelles have highly swollen cores in 1,4-dioxane. 1,4-dioxane was, however, preferred as a solvent compared to THF in the present study because of a better solubility of HNA in 1,4-dioxane.

Figure 1. (a) TEM images of micelles formed in 1,4-dioxane from the PS- $b$-P4VP copolymer; (b) the PS- $b$-P4VP/HNA mixture with a 4VP/HNA 1:0.25 molar ratio and (c) the PS- $b$-P4VP/HNA mixture with a 4VP/HNA 1:1 molar ratio.
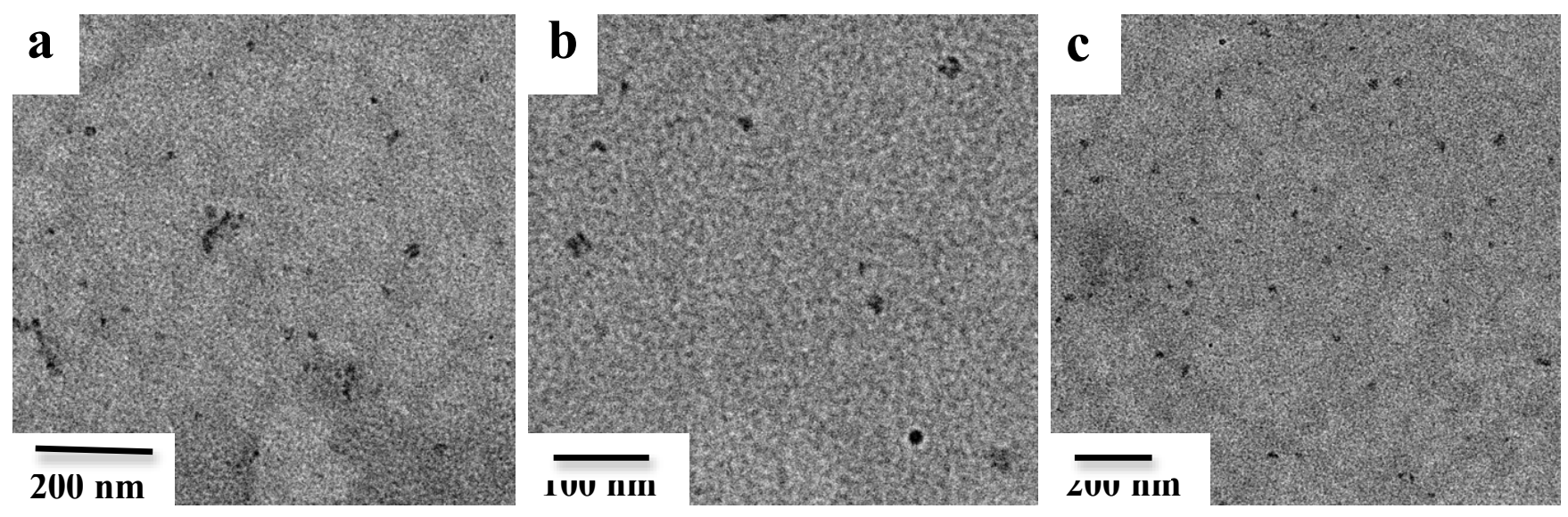

\subsection{Spectroscopic Investigations on Hydrogen-Bond Formation between PS-b-P4VP and HNA}

Before investigating the morphology of thin films prepared from the micellar solutions discussed in Section 3.1, the formation of hydrogen bonds between 4VP units and HNA molecules has been investigated by FTIR spectroscopy. Practically, FTIR spectra have been collected on thin films prepared from either the PS- $b$-P4VP starting copolymer or the PS- $b$-P4VP/HNA mixture with a 4VP/HNA 1:1 molar ratio. The PS- $b$-P4VP/HNA thin film has also been measured after removal of HNA molecules by methanol washing. Figure 2a shows the observed FTIR spectra between 1050 and $950 \mathrm{~cm}^{-1}$. The characteristic peak at $992 \mathrm{~cm}^{-1}$ corresponds to the free pyridine groups in the PS- $b$-P4VP block copolymer film (without added HNA). After addition of HNA, this peak shifts to $1009 \mathrm{~cm}^{-1}$ indicating hydrogen-bonded pyridine groups [19,37-39]. After washing with methanol, this peak again shifts to the initial value corresponding to the pure PS- $b$-P4VP block copolymer film, due to complete removal of the HNA from the P4VP + HNA phase. The formation of H-bonds is further evidenced by the characteristic peaks shown in Figure 2b, which clearly shows that those do form between the 
carboxylic acid groups of HNA and the P4VP blocks. Indeed, the characteristic carbonyl stretching frequency at $1674 \mathrm{~cm}^{-1}$ for HNA (corresponding to not-bound - $\mathrm{COOH}$ group) shifted to $1697 \mathrm{~cm}^{-1}$, because of hydrogen bond formation between the carboxylic acid groups and the pyridine moieties of the P4VP blocks [39-41]. Finally, the two broad bands observed at around $2500 \mathrm{~cm}^{-1}$ and $1960 \mathrm{~cm}^{-1}$ are the signature of strong H-bonding between HNA and P4VP [40,42].

In conclusion, FTIR spectroscopy clearly evidenced the formation of strong H-bonding between the carboxylic acid group of HNA molecules and the P4VP block of PS- $b$-P4VP copolymers. On the other hand, no clear contribution of the phenol group of HNA to hydrogen bonding has been observed. Those results are not surprising, since carboxylic acids are known to interact much more strongly with amino groups than with alcohols. At the limit, a partial proton transfer could occur from the carboxylic acid of HNA to the pyridine group of P4VP (acid-base reaction).

Figure 2. FTIR difference spectra of PS- $b$-P4VP, HNA, PS- $b$-P4VP/HNA mixture (4VP/HNA 1:1 molar ratio) and PS- $b$-P4VP/HNA mixtures after methanol washing.

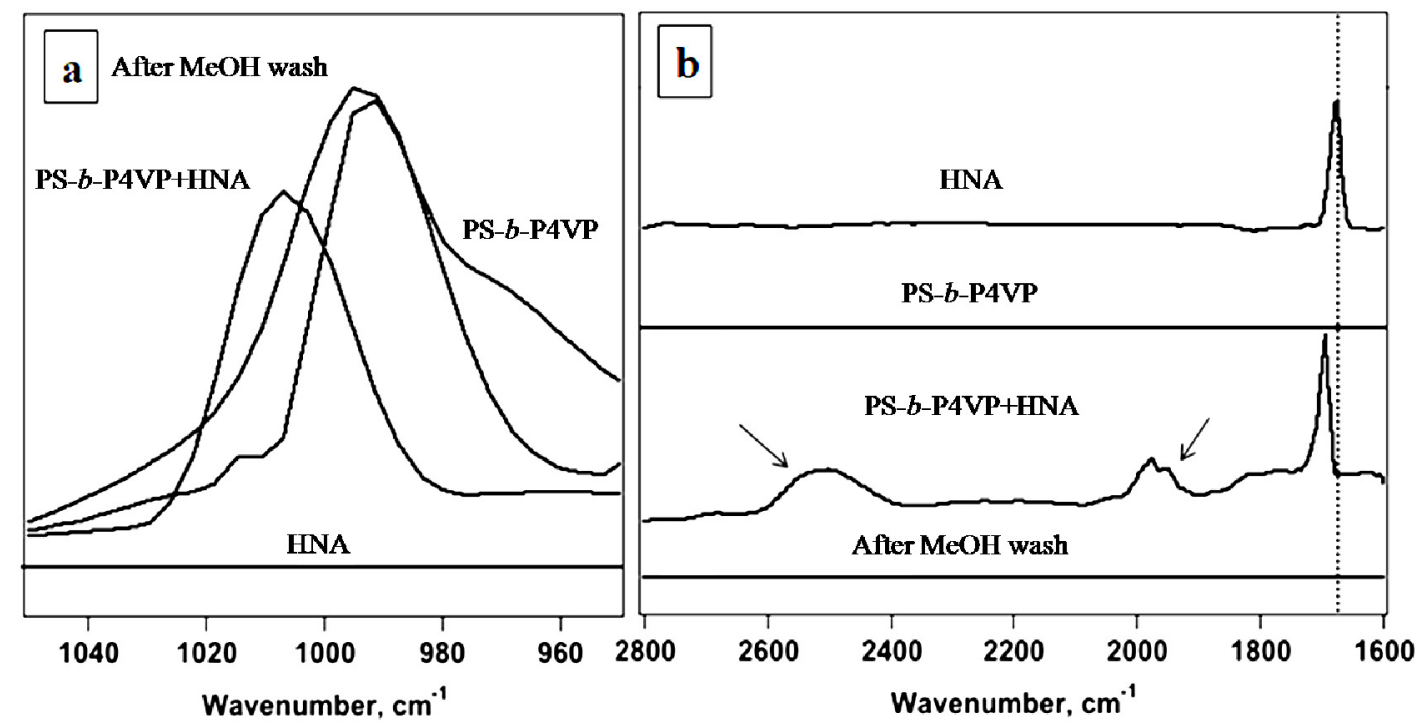

\subsection{Formation of Thin Films from PS-b-P4VP/HNA Mixtures}

In a first step, thin films have been prepared by spin-coating $1 \mathrm{wt} \%$ and $2 \mathrm{wt} \%$ solutions of the pure PS- $b$-P4VP copolymers in 1,4-dioxane and further annealing for $12 \mathrm{~h}$ in the same solvent before measurements (thin films before annealing showed an ill-defined morphology and will not be discussed here). After solvent annealing, microphase-separated dot-like nano-domains were clearly evidenced, as shown in Figure 3. Identical results have been obtained from either $1 \mathrm{wt} \%$ or $2 \mathrm{wt} \%$ solutions. Those results are in agreement with previously reported results on a similar system [37]. It is likely that the observed dot-like features in Figure 3 correspond to P4VP nano-domains dispersed in a PS matrix. The diameter of those nano-domains is found to be $18 \mathrm{~nm}$ and the inter-domain distance is $45 \mathrm{~nm}$. We hypothesize that the dot-like features observed in Figure 3 could be the apex of cylindrical, or at least elongated, P4VP nano-domains. 
Figure 3. AFM images (left: height image, right: phase image) of a PS- $b$-P4VP thin film spin-coated on a silicon substrate from a $2 \mathrm{wt} \%$ solution in 1,4-dioxane (images are $2 \times 2 \mu \mathrm{m}^{2}$ ).

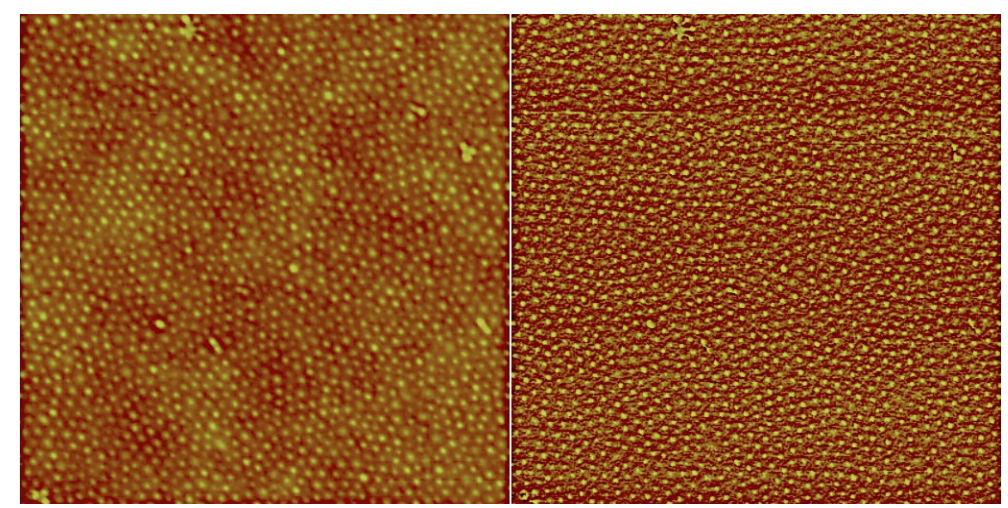

It is well known that the orientation of cylindrical nano-domains in block copolymer thin films is strongly dependent on sample preparation procedures and can be tuned when proper solvent annealing conditions are applied. The preferential orientation of the P4VP nano-domains can be attributed, on one hand, to the selectivity of the constituted blocks in the solvent used for solution preparation and annealing. 1,4-Dioxane is indeed a selective solvent for PS, but not for P4VP [11]. So, annealing in 1,4-dioxane will induce a perpendicular orientation of the P4VP nano-domains, in agreement with previously reported results [11]. On the other hand, specific interactions between the substrate and the constituent polymer blocks have also to be taken into account. In our case, specific interactions via hydrogen bonding are expected to occur between the silanol groups located at the surface of the used silicon wafer and the P4VP block of the spin-coated PS- $b$-P4VP block copolymer. The use of 1,4-dioxane as a solvent here is critical, since this solvent is a H-bond acceptor capable of interacting with the silanol groups of the substrate at the expense of the P4VP blocks. Therefore, 1,4-dioxane allows the neutralization of specific interactions between the substrate and the spin-coated thin films. Taking into account the limited thickness of the thin films prepared in this study on a "neutralized" substrate, the P4VP nano-domains have, thus, the tendency to orient perpendicularly [17,43].

In a following step, thin films have been prepared by spin-coating a $1 \mathrm{wt} \%$ solution of the PS- $b$-P4VP/HNA mixture with a 4VP/HNA 1:1 molar ratio in 1,4-dioxane (Figure 4). In contrast to the PS- $b$-P4VP thin films, well-defined nanodomains have been observed for the as-spun thin films. Annealing for $12 \mathrm{~h}$ in 1,4-dioxane led to a nanostructured thin film similar to the one obtained for the PS- $b$-P4VP system (compare Figures 3 and $4 b$ ).

It is remarkable to notice that the diameter of the dot-like features mostly increased (from 18 to $28 \mathrm{~nm}$ ) compared to the inter-dot distance (from 48 to $52 \mathrm{~nm}$ ) when the PS- $b$-P4VP and PS- $b$-P4VP/HNA systems annealed in 1,4-dioxane are compared. Therefore, it is clear that the HNA molecules essentially swell the P4VP nanodomains. The dot-like features can be again ascribed as the apex of perpendicularly oriented P4VP/HNA cylindrical nanodomains embedded in a PS matrix. It should be, however, noted that the volume fraction of the $\mathrm{P} 4 \mathrm{VP} / \mathrm{HNA}$ microphase in the investigated PS- $b$-P4VP/HNA mixture is roughly estimated to be 0.48 . Thus, a lamellar morphology is expected for the nanostructures formed in PS- $b$-P4VP/HNA thin films [44], which is obviously not the case. Since 1,4-dioxane is a much better solvent for PS than for P4VP/HNA, the PS matrix swells much more than the P4VP/HNA nano-domains during the solvent annealing process. The swelled PS could maintain the system in the 
cylindrical morphology region of the phase diagram and prevent the formation of the expected lamellar morphology. Since the drying of the film after the annealing is a rather fast process, this could lead to the freezing of this non-equilibrium morphology in the thin films prepared from the PS- $b$-P4VP/HNA mixture.

Figure 4. AFM images (both height and phase images are displayed) for thin films prepared from the PS- $b$-P4VP/HNA mixture (4VP/HNA 1:1 molar ratio) as (a) spun and (b) annealed in 1,4-dioxane (images are $2 \times 2 \mu \mathrm{m}^{2}$ ).
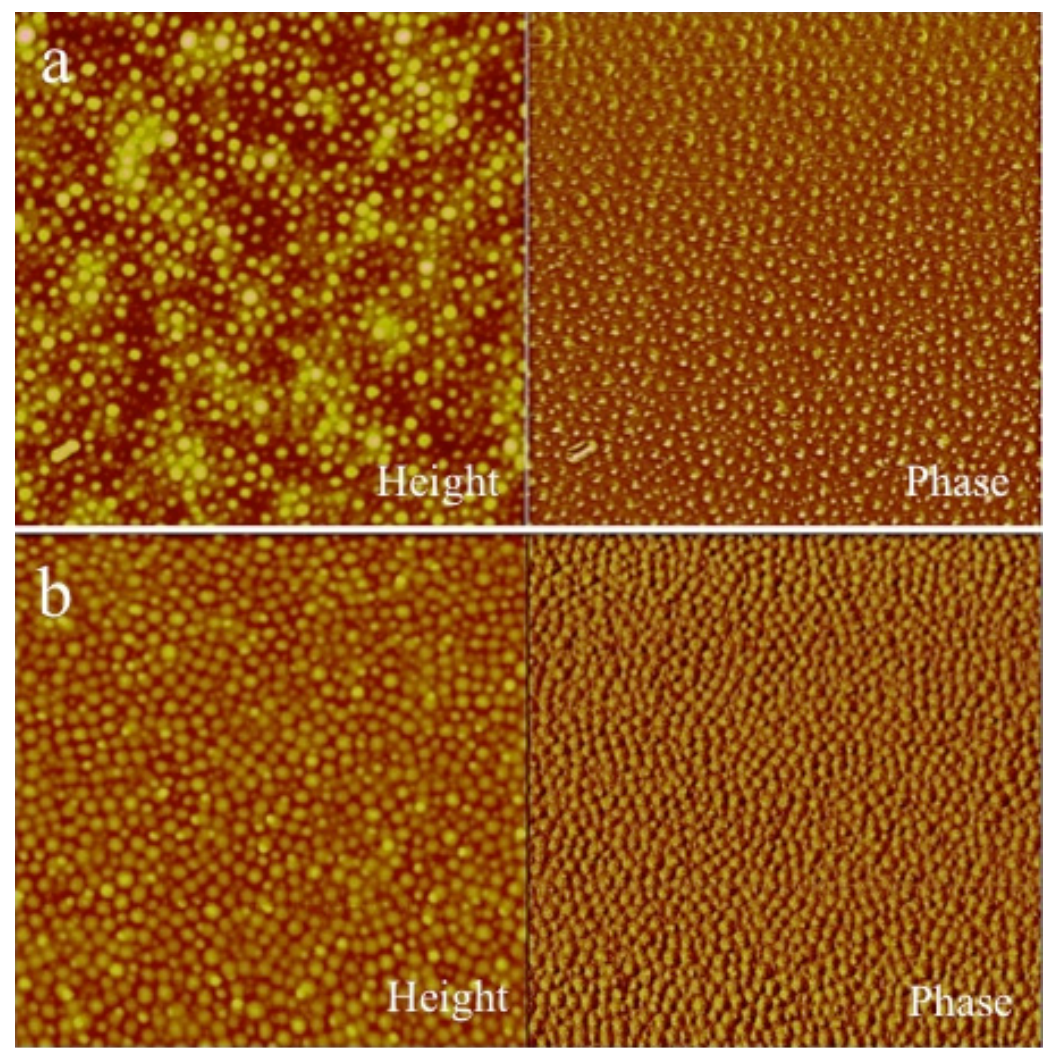

At this stage, it should be recalled that micellar structures are formed by the PS- $b$-P4VP/HNA mixture in 1,4-dioxane. Thus, the following mechanism could be tentatively proposed to explain the nanostructures observed in Figure 4. In the first step, the PS- $b$-P4VP/HNA spherical micelles would be deposited on the substrate by the spin-coating process. This would result in a thin layer of de-organized micelles, as visualized in Figure 4a, in which the bright dot-like features likely correspond to P4VP/HNA micellar cores. Further annealing of those thin films in 1,4-dioxane would lead to a reorganization of those micelles, which would tend to stack and merge to ultimately form a hexagonal array of P4VP/HNA cylinders oriented perpendicular to the film surface. Due to the limited thickness of the thin films investigated here $(\mathrm{ca} .50 \mathrm{~nm})$ compared to the diameter of the P4VP/HNA nanodomains $(28 \mathrm{~nm})$, the latter can be considered dots rather than real cylinders. The expected thermodynamic state for this system (a lamellar morphology according to the composition of the PS- $b$-P4VP/HNA blend) would, however, have never been reached (at least under the explored experimental parameters in this study), confirming the formation of out-of-equilibrium nanostructures strongly dependent on the experimental conditions.

The effect of the solvent used for annealing has been investigated, too. Indeed, the PS- $b$-P4VP/HNA thin films have been annealed either in THF or 1,4-dioxane for $24 \mathrm{~h}$. THF has been selected, since this solvent was used by Bazuin and co-workers for the preparation of thin films from PS- $b$-P4VP mixed 
with naphthol or naphthoic acid [37]. Although both solvents are rather similar in term of chemical structure (both are ethers), only 1,4-dioxane allowed the reorganization of the originally spun micelles into a cylindrical thin films (Figure 4b), while the thin films annealed in THF (data not shown) essentially showed the as-spun micelles of Figure $4 \mathrm{a}$. This can be understood by the fact that 1,4-dioxane was experimentally observed to be a better solvent for HNA molecules and, therefore, favors reorganization of the P4VP/HNA domains compared to THF.

The effect of the concentration of the starting PS- $b$-P4VP/HNA micellar solution was also investigated. A PS- $b$-P4VP/HNA solution at a concentration of $2 \mathrm{wt} \%$ in 1,4-dioxane was spin-coated onto the silicon substrate. It has been previously shown that PS- $b$-P4VP/HNA spherical micelles are formed in solution at this concentration (see Section 3.1). However, worm-like features were directly observed after spin-coating those solutions (Figure 5), suggesting that the initial spherical micelles had already merged at this stage into cylindrical micelles. Those worm-like features were not well ordered on the substrate and were mainly oriented parallel to it. Further annealing was realized either in THF or 1,4-dioxane for $12 \mathrm{~h}$. Again, annealing in THF was not successful, as the same morphology as the one depicted in Figure 5 was observed. In contrast, annealing in 1,4-dioxane resulted in the formation of a thin film showing a dense array of perpendicular cylinders similar to Figure $4 \mathrm{~b}$ (data not shown). It should be noted that the thin films prepared from 2 wt \% solutions displayed almost a double thickness compared to the analogues prepared from $1 \mathrm{wt} \%$ solutions. Obviously, more material is deposited on the substrate when the concentration of the starting PS- $b$-P4VP/HNA solution is increased. Therefore, it seems logical that the structures resulting from the merging of the spherical micelles during the spin-coating process have the opportunity to elongate towards short worms when the concentration of the micellar solution is increased.

The effect of the 4VP/HNA molar ratio on the morphology of the PS- $b$-P4VP/HNA thin films has been also investigated. Basically, similar results as the ones depicted in Figure 4 have been observed before and after annealing in 1,4-dioxane for thin films prepared for 1:0.25, 1:0.5, 1:0.75 and 1:1 4VP/HNA molar ratios. The inter-dot distance was found to be equal to $c a .50 \mathrm{~nm}$, whatever the 4VP/HNA molar ratio. However, the dot diameters with values in the range of 25 to $28 \mathrm{~nm}$ were slightly greater than for the pure PS- $b$-P4VP thin films, confirming the swelling of the P4VP domains with HNA molecules. Due to experimental uncertainties in those AFM measurements, it is, however, not possible to establish a relationship between dot diameter and 4VP/HNA ratio.

Figure 5. AFM height (a) and phase (b) images for thin films prepared from the PS- $b$-P4VP/HNA mixture (4VP/HNA 1:1 molar ratio) at a concentration of $2 \mathrm{wt} \%$ in 1,4-dioxane (images are $3 \times 3 \mu^{2}$ ).

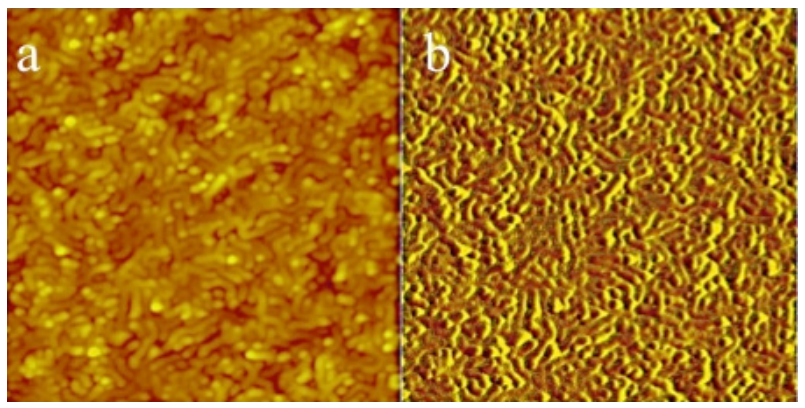


FTIR spectroscopy revealed essentially the formation of hydrogen bonds between the carboxylic acid groups of HNA and 4VP units. This suggests that hydroxyl groups on HNA molecules are still available and free to react with other molecules. The use of HNA could be seen as a way to impart a hierarchy of interactions in our system. In order to demonstrate this possibility, we have immersed the PS- $b$-P4VP/HNA thin films prepared from 1 and $2 \mathrm{wt} \%$ solutions in 1,4-dioxane, in an aqueous solution of borax $\left(\mathrm{Na}_{2} \mathrm{~B}_{4} \mathrm{O}_{7}\right)$. Indeed, borax is well known for forming boronic esters with hydroxyl groups (1 borax molecule can react with up to four hydroxyl groups), leading to cross-linked materials and gels. In this respect, gels have been already described from borax/poly(vinyl alcohol)/naphthol mixtures [45]. Therefore, we believe that borax would be able to cross-link the P4VP/HNA nano-domains by first penetrating into them and secondly reacting with the free hydroxyl groups of HNA molecules. In order to demonstrate that the P4VP/HNA nano-domains have been indeed cross-linked after immersion in a slightly acidic borax solution, the thin films have been immersed in 1,4-dioxane for an extended time. This process is expected to detach (part of) the PS- $b$-P4VP/HNA thin films that could be further re-solvated and could release PS- $b$-P4VP/HNA micelles in solution. Four samples have been investigated following this methodology: the non-cross-linked thin film prepared from the $1 \mathrm{wt} \% \mathrm{PS}-b$-P4VP/HNA solution, the non-cross-linked thin film prepared from the $2 \mathrm{wt} \% \mathrm{PS}-b$-P4VP/HNA solution, the borax cross-linked thin film prepared from the 1 wt $\%$ PS- $b$-P4VP/HNA solution and the borax cross-linked thin film prepared from the $2 \mathrm{wt} \% \mathrm{PS}-b$-P4VP/HNA solution. The resulting solutions have been then analyzed by DLS in order to detect the eventual presence of micelles in 1,4-dioxane. For the two first samples, micelles with $R_{\mathrm{h}}$ 's of $c a .40 \mathrm{~nm}$ were detected. Those values are in agreement with the $R_{\mathrm{h}}$ observed for the original PS- $b$-P4VP/HNA micelles before film preparation (see Section 3.1). In contrast, larger micelles were detected for the two borax cross-linked samples, i.e., $R_{\mathrm{h}}$ of 62 and $115 \mathrm{~nm}$ for the borax cross-linked thin film prepared from the 1 and $2 \mathrm{wt} \% \mathrm{PS}-b$-P4VP/HNA solutions, respectively. Those $R_{\mathrm{h}}$ values roughly correspond to the thickness of the respective PS- $b$-P4VP/HNA thin films and give credit to the formation of borax cross-linked P4VP/HNA domains that could not be reorganized once they are re-dispersed in solution. The whole process leading to the formation of those stabilized borax cross-linked micelles is depicted in Scheme 2.

In a final experiment, the PS- $b$-P4VP/HNA thin films annealed in 1,4-dioxane have been extensively washed with methanol, a good solvent for HNA and P4VP and a bad solvent for PS. According to FTIR results previously discussed in Section 3.2, this process allows the disruption of hydrogen bonds between HNA molecules and P4VP and removal of the HNA molecules from the P4VP/HNA cylinders in the thin films. The FTIR results have been complemented by AFM imaging in the dried state, as shown in Figure 6. By comparing both height and phase contrast images of the thin film before (Figure 4b) and after removal of HNA molecules (Figure 6), one can notice that the contrasts in height and phase have been inverted. Although this cannot be considered as a definitive proof of the formation of a porous material, this observation indicates that something changed in the thin film after HNA extraction. Moreover, the AFM image of Figure 6 shows strong similarities with previously reported results on a similar porous system [18]. The analysis of the AFM image in Figure 6 revealed an average pore diameter of $20 \mathrm{~nm}$ and an inter-pore distance of $49 \mathrm{~nm}$. Those values are intermediate compared to the pure PS- $b$-P4VP (18 and $48 \mathrm{~nm}$, respectively) and the PS- $b$-P4VP/HNA thin films (28 and $52 \mathrm{~nm}$, respectively). Taking into account that AFM imaging has been realized in the dried state, one can consider that the AFM images shown in Figure 6 correspond to a nanoporous thin film in which the continuous matrix is formed by 
the PS blocks plus the P4VP blocks that are collapsed on the pore walls as a result of the drying process. Following this hypothesis, the dot-like features observed in Figure 6 would be voids.

Scheme 2. Proposed mechanism for the formation of nanostructured thin films from PS- $b$-P4VP/HNA micelles in 1,4-dioxane, followed by the cross-linking of P4VP/HNA nano-domains by $\mathrm{Na}_{2} \mathrm{~B}_{4} \mathrm{O}_{7}$ and the further release of micelles with cross-linked cores.

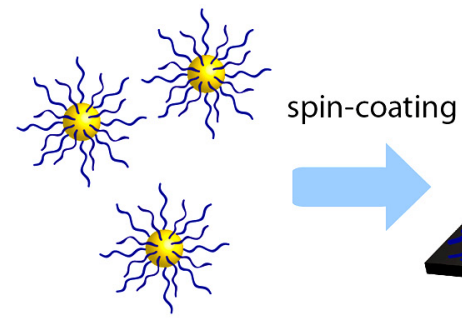

micelles with P4VP/HNA cores and PS corona in 1,4-dioxane

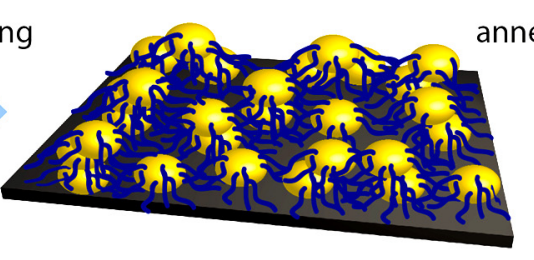

layer of spin-coated micelles on a substrate annealing in 1,4-dioxane

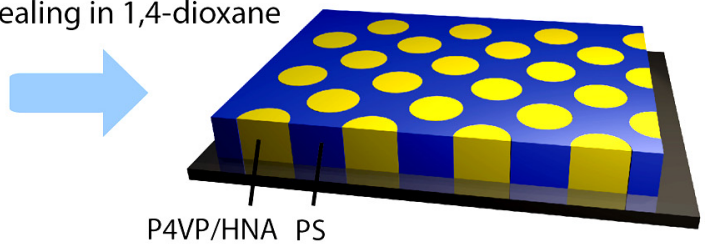

Thin film with an hexagonal array of P4VP/HNA nanodomains in a PS matrix

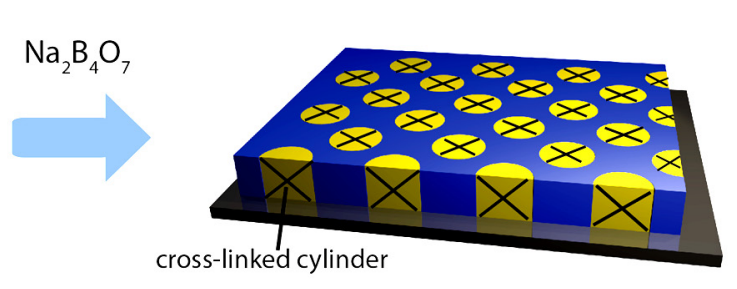

Thin film with an hexagonal array of cross-linked P4VP/HNA nanodomains

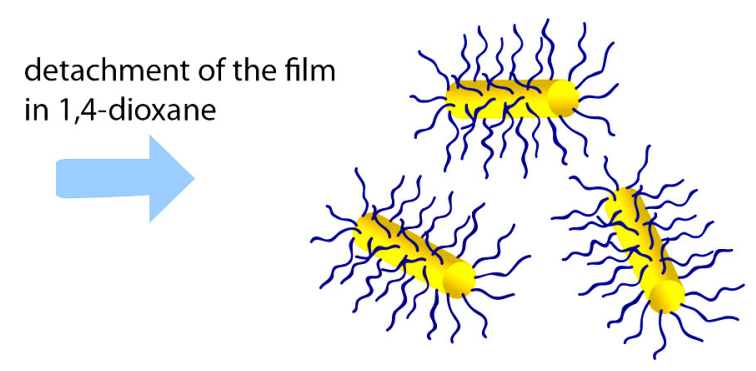

micelles with cross-linked P4VP/HNA cores

Figure 6. AFM height and phase images for a nanoporous thin film prepared from the PS- $b$-P4VP/HNA mixture (4VP/HNA 1:1 molar ratio) at $1 \mathrm{wt} \%$ in 1,4-dioxane, annealed for $12 \mathrm{~h}$ in 1,4-dioxane and, finally, rinsed with methanol to remove HNA molecules (images are $2 \times 2 \mu \mathrm{m}^{2}$ ).

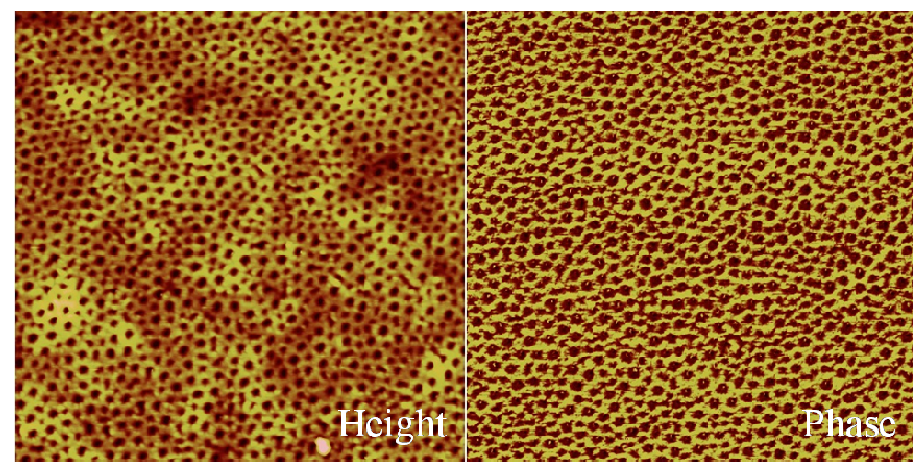

\section{Conclusions}

In this paper, non-equilibrium nanostructures have been prepared from PS- $b$-P4VP diblock copolymers blended with HNA small molecules. Hydrogen-bonding between the 4VP units and the carboxylic group of HNA has been evidenced by FTIR spectroscopy. The PS- $b$-P4VP/HNA complexes form spherical micelles in 1,4-dioxane, consisting of a P4VP/HNA core surrounded by PS coronal 
chains. Those micelles are similar to the ones formed by the PS- $b$-P4VP alone in the same solvent, and a comparison between DLS and TEM results strongly suggests that the micellar cores are highly swollen in both cases. Those results are in line with previously reported results on a related system [37]. Thin films have been prepared by spin-coating those micellar solutions on a substrate. After spin-coating, a layer of micelles is observed on the substrate that transforms into an ordered thin film constituted of P4VP/HNA cylinders perpendicularly oriented to the film surface and hexagonally arranged in the PS matrix after annealing in 1,4-dioxane. Those cylinders are thought to originate from the merging of the spherical micelles present in solution. Compared to the thin films prepared from the pure PS- $b$-P4VP copolymer that also show a cylindrical morphology, the ones from the PS- $b$-P4VP/HNA mixtures show larger nanodomains, due to the presence of HNA molecules. Washing with methanol finally allows one to get rid of HNA molecules inside the nanodomains and to create nanoporosity.

Finally, comparison of our findings with previously reported results from Bazuin and co-workers [37-39] confirms that the interaction of carboxylic groups with 4VP units is stronger than hydrogen bonding between hydroxyl groups and 4VP. Direct comparison of the thin film morphologies in both studies is, however, not possible, since different methods of preparation have been used (spin-coating by us and dip-coating at different rates for Bazuin and coworkers). Comparison with the results previously reported by Stamm and coworkers is also interesting [17,18]. Those authors demonstrated that it is possible to finely tune the orientation of the nano-domains in the thin films obtained by spin-coating of PS- $b$-P4VP/HABA mixtures by using proper annealing conditions. They also showed the possibility to create nanoporous materials by selective extraction of the HABA molecules. Although HABA is a difunctional molecule containing both carboxylic acid and hydroxyl functionalities, they did not investigate the possibility to introduce a hierarchy of interactions in the accordingly obtained materials. It is also very interesting to compare our findings with the recent results of $\mathrm{Wu}$ and Bubeck, who compared the effect of annealing in 1,4-dioxane for thin films prepared either from a carboxylic acid bearing a small molecule and PS- $b$-P4VP or from a hydroxyl functionalized small molecule and the same PS- $b$-P4VP block copolymer [46]. Macrophase separation was observed after annealing of the carboxylic acid small molecule/PS- $b$-P4VP thin film, while microphase separation was maintained for the annealed hydroxyl functionalized small molecule/PS- $b$-P4VP thin film. Macrophase separation was attributed to the disruption of the carboxylic acid/P4VP H-bonds at the expense of H-bonds driven dimerization of carboxylic acid small molecules, which further phase separate and crystallize on top of the thin film. No macrophase separation was observed for the PS- $b$-P4VP/HNA thin films annealed in 1,4-dioxane investigated in this study. This could be attributed to the beneficial use of a small molecule containing, at the same time, the carboxylic acid and hydroxyl functionalities, the latter preventing macrophase separation to occur.

In this report, the free hydroxyl groups of HNA have been used not only to prevent macrophase separation during annealing in 1,4-dioxane, but also to be cross-linked by borax, which further allows stabilization of the P4VP/HNA domains, which can be further detached from the substrate and re-dispersed in solution as micelles. Our approach based on a difunctional molecule with distinct functionalities, therefore, allows the introduction of a hierarchy of interactions in the accordingly obtained materials. This significantly broadens the scope of applications of such materials. 


\section{Acknowledgements}

B.B. and J.F.G. are grateful to BELSPO for a post-doc grant in the frame of the IAP project P7/05 Functional Supramolecular Systems and to the P2M Programme from the ESF. E.P. is grateful to FRIA for financial support.

\section{Conflict of interest}

The authors declare no conflict of interest.

\section{References}

1. Hamley, I.W. The Physics of Block Copolymers; Oxford University Press: New York, NY, USA, 1998.

2. Bates, F.S.; Fredrickson, G.H. Block copolymers-designer soft materials. Phys. Today 1999, 52, 32-38.

3. Krausch, G.; Magerle, R. Nanostructured thin films via self-assembly of block copolymers. Adv. Mater. 2002, 14, 1579-1583.

4. Bates, F.S.; Fredrickson, G.H. Block copolymer thermodynamics: Theory and experiments. Annu. Rev. Phys. Chem. 1990, 41, 525-557.

5. Thurn-Albrecht, T.; Schotter, J.; Kastle, G.A.; Emley, N.; Shibauchi, T.; Krusin-Elbaum, L.; Guarini, K.; Black, C.T.; Tuominen, M.T.; Russell, T.P. Ultrahigh-density nanowire arrays grown in self-assembled diblock copolymer templates. Science 2000, 290, 2126-2129.

6. Ramanathan, M.; Nettleton, E.; Darling, S.B. Simple orientational control over cylindrical organic-inorganic block copolymer domains for etch mask applications. Thin Solid Films 2009, 517, 4474-4478.

7. Knoll, A.; Horvat, A.; Lyakhova, K.S.; Krausch, G.; Sevink, G.J.A.; Zvelindovsky, A.V.; Magerle, R. Phase behavior in thin films of cylinder-forming block copolymers. Phys. Rev. Lett. 2002, 89, 35501-35504.

8. Park, M.; Harrison, C.; Chaikin, P.M.; Register, R.A.; Adamson, D.H. Block copolymer lithography: Periodic arrays of $\sim 10^{11}$ holes in 1 square centimeter. Science 1997, 276, 1401-1404.

9. Haberkorn, N.; Lechmann, M.C.; Sohn, B.H.; Char, K.; Gutmann, J.S.; Theato, P. Templated organic and hybrid materials for optoelectronic applications. Macromol. Rapid Commun. 2009, 30, 1146-1166.

10. Lo, K.H.; Chen, M.C.; Ho, R.M.; Sung, H.W. Pore-filling nanoporous templates from degradable block copolymers for nanoscale drug delivery. ACS Nano 2009, 3, 2660-2666.

11. Kuila, B.K.; Gowd, E.B.; Stamm, M. Supramolecular assembly of poly(styrene)- $b$-poly (4-vinylpyridine) and 1-pyrenebutyric acid in thin film and their use for nanofabrication. Macromolecules 2010, 43, 7713-7721.

12. Kuila, B.K.; Nandan, B.; Bohme, M.; Janke, A.; Stamm, M. Vertically oriented arrays of polyaniline nanorods and their super electrochemical properties. Chem. Commun. 2009, 5749-5751. 
13. Kuila, B.K.; Stamm, M. Fabrication of oriented polyaniline nanostructures using block copolymer nanotemplates and their optical, electrochemical and electric properties. J. Mater. Chem. 2010, 20, 6086-6094.

14. Gohy, J.F.; Mores, S.; Varshney, S.K.; Jerome, R. Self-organization of water-soluble complexes of a poly(2-vinylpyridinium)-block-poly(ethylene oxide) diblock with fluorinated anionic surfactants. Macromolecules 2003, 36, 2579-2581.

15. Ruokolainen, J.; Saariaho, M.; Ikkala, O.; ten Brinke, G.; Thomas, E.L.; Torkkeli, M.; Serimaa, R. Supramolecular routes to hierarchical structures: Comb-coil diblock copolymers organized with two length scales. Macromolecules 1999, 32, 1152-1158.

16. Ikkala, O.; ten Brinke, G. Hierarchical self-assembly in polymeric complexes: Towards functional materials. Chem. Commun. 2004, 2131-2137.

17. Tokarev, I.; Krenek, R.; Burkov, Y.; Schmeisser, D.; Sidorenko, A.; Minko, S.; Stamm, M. Microphase separation in thin films of poly(styrene-block-4-vinylpyridine) copolymer-2-(4'hydroxybenzeneazo) benzoic acid assembly. Macromolecules 2005, 38, 507-516.

18. Sidorenko, A.; Tokarev, I.; Minko, S.; Stamm, M. Ordered reactive nanomembranes/nanotemplates from thin films of block copolymer supramolecular assembly. J. Am. Chem. Soc. 2003, 125, 12211-12216.

19. Laforgue, A.; Bazuin, C.G.; Prud'homme, R.E. A study of the supramolecular approach in controlling diblock copolymer nanopatterning and nanoporosity on surfaces. Macromolecules 2006, 39, 6473-6482.

20. Tang, C.; Hur, S.; Stahl, B.C.; Sivanandan, K.; Dimitriou, M.; Pressly, E.; Fredrickson, G.H.; Kramer, E.J.; Hawker, C.J. Thin film morphology of block copolymer blends with tunable supramolecular interactions for lithographic applications. Macromolecules 2010, 43, 2880-2889.

21. Tung, S.H.; Kalarickal, N.C.; Mays, J.W.; Xu, T. Hierarchical assemblies of block-copolymerbased supramolecules in thin films. Macromolecules 2008, 41, 6453-6462.

22. Ruokolainen, J.; Tanner, J.; ten Brinke, G.; Ikkala, O.; Torkelli, M.; Serimaa, R. Poly(4-vinyl pyridine)/zinc dodecyl benzene sulfonate mesomorphic state due to coordination complexation. Macromolecules 1995, 28, 7779-7784.

23. Wood, K.C.; Little, S.R.; Langer, R.; Hammond, P.T. A family of hierarchically self-assembling linear-dendritic hybrid polymers for highly efficient targeted gene delivery. Angew. Chem. Int. Ed. 2005, 44, 6704-6708.

24. Faul, C.F.; Antonietti, M. Ionic self-assembly: Facile synthesis of supramolecular materials. Adv. Mater. 2003, 15, 673-683.

25. Thunemann, A.F. Polyelectrolyte-surfactant complexes (synthesis, structure and materials aspects). Prog. Polym. Sci. 2002, 27, 1473-1572.

26. Van Ekenstein, G.A.; Polushkin, E.; Nijland, H.; Ikkala, O.; ten Brinke, G. Shear alignment at two length scales: Comb-shaped supramolecules self-organized as cylinders-within-lamellar hierarchy. Macromolecules 2003, 36, 3684-3688.

27. Kosonen, H.; Ruokolainen, J.; Knaapila, M.; Torkkeli, M.; Serimaa, R.; Bras, W.; Monkman, A.P.; ten Brinke, G.; Ikkala, O. Self-organized nanostructures of conducting polyaniline and hydrogen bonded amphiphiles. Synth. Met. 2001, 121, 1277-1278. 
28. Xuan, Y.; Peng, J.; Cui, L.; Wang, H.F.; Li, B.Y.; Han, Y.C. Morphology development of ultrathin symmetric diblock copolymer film via solvent vapor treatment. Macromolecules 2004, 37, 7301-7307.

29. Elbs, H.; Drummer, C.; Abetz, V.; Krausch, G. Thin film morphologies of ABC triblock copolymers prepared from solution. Macromolecules 2002, 35, 5570-5577.

30. Bosworth, J.K.; Paik, M.Y.; Ruiz, R.; Schwartz, E.L.; Huang, J.; Ko, A.W.; Smilgies, D.M.; Black, C.T.; Ober, C.K. Control of self-assembly of lithographically patternable block copolymer films. ACS Nano 2008, 2, 1396-1402.

31. Huang, W.; Chen, P.Y.; Tung, S.H. Effects of annealing solvents on the morphology of block copolymer-based supramolecular thin films. Macromolecules 2012, 45, 1562-1569.

32. Van Zoelen, W.; Asumaa, T.; Ruokolainen, J.; Ikkala, O.; ten Brinke, G. Phase behavior of solvent vapor annealed thin films of PS-b-P4VP(PDP) supramolecules. Macromolecules 2008, 41, 3199-3208.

33. Van Zoelen, W.; Bondzic, S.; Landaluce, T.F.; Brondijk, J.; Loos, K.; Schouten, A.J.; Rudolph, P.; ten Brinke, G. Nanostructured polystyrene-block-poly(4-vinyl pyridine)(pentadecylphenol) thin films as templates for polypyrrole synthesis. Polymer 2009, 50, 3617-3625.

34. Seifarth, O.; Krenek, R.; Tokarev, I.; Burkov, Y.; Sidorenko, A.; Minko, S.; Stamm, M.; Schmeisser, D. Metallic nickel nanorod arrays embedded into ordered block copolymer templates. Thin Solid Films 2007, 515, 6552-6556.

35. Bohme, M.; Kuila, B.; Schlorb, H.; Nandan, B.; Stamm, M. Thin films of block copolymer supramolecular assemblies: Microphase separation and nanofabrication. Phys. Status Solidi B 2010, 247, 2458-2469.

36. Nandan, B.; Vyas, M.K.; Bohme, M.; Stamm, M. Composition-dependent morphological transitions and pathways in switching of fine structure in thin films of block copolymer supramolecular assemblies. Macromolecules 2010, 43, 2463-2473.

37. Roland, S.; Gaspard, D.; Prud'homme, R.E.; Bazuin, C.G. Morphology evolution in slowly dipcoated supramolecular PS- $b$-P4VP thin films. Macromolecules 2012, 45, 5463-5476.

38. Roland, S.; Prud'homme, R.E.; Bazuin, C.G. Morphology, thickness, and composition evolution in supramolecular block copolymer films over a wide range of dip-coating rates. ACS Macro Lett. 2012, 1, 973-976.

39. Roland, S.; Pellerin, C.; Bazuin, C.G.; Prud'homme, R.E. Evolution of small molecule content and morphology with dip-coating rate in supramolecular PS-P4VP thin films. Macromolecules 2012, 45, 7964-7972.

40. Brandys, F.A.; Bazuin, C.G. Mixtures of an acid-functionalized mesogen with poly(4-vinylpyridine). Chem. Mater. 1996, 8, 83-92.

41. Lee, J.Y.; Painter, P.C.; Coleman, M. Hydrogen bonding in polymer blends. 4. Blends involving polymers containing methacrylic acid and vinylpyridine groups. Macromolecules 1988, 21, 954-960.

42. Kato, T.; Kihara, H.; Uryu, T.; Fujishima, A.; Fréchet, J. Molecular self-assembly of liquid crystalline side-chain polymers through intermolecular hydrogen bonding-Polymeric complexes built from a polyacrylate and stilbazoles. Macromolecules 1992, 25, 6836-6841.

43. Kim, S.H.; Misner, M.J.; Xu, T.; Kimura, M.; Russell, T.P. Highly oriented and ordered arrays from block copolymers via solvent evaporation. Adv. Mater. 2004, 16, 226-231. 
44. Ruokolainen, J.; ten Brinke, G.; Ikkala, O. Supramolecular polymeric materials with hierarchical structure-within-structure morphologies. Adv. Mater. 1999, 11, 777-780.

45. Lee, S.M.; Chung, W.Y.; Kim, J.K.; Suh, D.H. A novel fluorescence temperature sensor based on a surfactant-free PVA/borax/2-naphthol hydrogel network system. J. Appl. Polym. Sci. 2004, 93, 2114-2118.

46. Wu, S.; Bubeck, C. Macro- and microphase separation in block copolymer supramolecular assemblies induced by solvent annealing. Macromolecules 2013, 46, 3512-3518.

(C) 2013 by the authors; licensee MDPI, Basel, Switzerland. This article is an open access article distributed under the terms and conditions of the Creative Commons Attribution license (http://creativecommons.org/licenses/by/3.0/). 